\title{
Influenza Vaccination in Older Adults: Recent Innovations and Practical Applications
}

\author{
Melissa K. Andrew ${ }^{1,2}$. Susan K. Bowles ${ }^{1,2,3} \cdot$ Graham Pawelec $^{4,5} \cdot$ Laura Haynes $^{6} \cdot$ George A. Kuchel $^{6}$. \\ Shelly A. McNeil ${ }^{2,7}$. Janet E. McElhaney ${ }^{8}$
}

Published online: 9 November 2018

(c) Springer Nature Switzerland AG 2018

\begin{abstract}
Influenza can lead to serious illness, particularly for older adults. In addition to short-term morbidity and mortality during the acute infection, recovery can be prolonged and often incomplete. This may lead to persistent declines in health and function, including catastrophic disability, which has dramatic implications for the well-being and support needs of older adults and their caregivers. All of this means that prevention of infection and effective treatment when illness has occurred are of paramount importance. In this narrative review, we discuss the effectiveness of influenza vaccines for the prevention of influenza illness and serious outcomes in older adults. We review evidence of vaccine effectiveness for older adults in comparison with younger age groups, and also highlight the importance of frailty as a determinant of vaccine effectiveness. We then turn our attention to the question of why older and frailer individuals have poorer vaccine responses, and consider changes in immune function and inflammatory responses. This sets the stage for a discussion of newer influenza vaccine products that have been developed with the aim of enhancing vaccine effectiveness in older adults. We review the available evidence on vaccine efficacy, effectiveness and cost benefits, consider the potential place of these innovations in clinical geriatric practice, and discuss international advisory committee recommendations on influenza vaccination in older adults. Finally, we highlight the importance of influenza prevention to support healthy aging, along with the need to improve vaccine coverage rates using available vaccine products, and to spur development of better influenza vaccines for older adults in the near future.
\end{abstract}

Melissa K. Andrew

mandrew@dal.ca

1 Division of Geriatric Medicine, Department of Medicine (Geriatrics), Dalhousie University, 5955 Veterans' Memorial Lane, Halifax, NS, Canada

2 Canadian Center for Vaccinology, Halifax, NS, Canada

3 Department of Pharmacy, Nova Scotia Health Authority, Central Zone, Halifax, NS, Canada

4 Second Department of Internal Medicine, University of Tübingen, Tübingen, Germany

5 Health Sciences North Research Institute, Sudbury, ON, Canada

6 Center on Aging, University of Connecticut School of Medicine, Farmington, CT, USA

7 Department of Medicine (Infectious Diseases), Dalhousie University, Halifax, NS, Canada

8 Health Sciences North Research Institute, Northern Ontario School of Medicine, Sudbury, ON, Canada

\section{Key Points}

Older adults are particularly vulnerable to poor outcomes from influenza over both short- and long-term time horizons.

Although immune responses generally decline with age, the prevention of influenza with vaccination is an important strategy to support healthy aging.

Several vaccine products are available for older adults, including standard-dose trivalent and quadrivalent vaccines, high-dose vaccine, adjuvanted vaccine, and recombinant vaccine.

Improving vaccine coverage rates using available vaccine products is an important goal. 


\section{Introduction}

\subsection{Influenza Can Have a Severe and Lasting Impact on Older Adults' Health and Well-Being}

Older adults are disproportionately affected by influenza and its complications. In the short-term (at the time of the acute infection), morbidity and mortality are significant problems [1]. Worldwide, it is estimated that $291,243-645,843$ people die from influenza and its respiratory complications each year, of whom older adults age $75+$ years are the most at-risk age group, with 51.3-99.4 deaths per 100,000 people aged $75+$ years versus $13.3-27.8 / 100,000$ for ages $65-74$ years and $1.0-5.1 / 100,000$ for those $<65$ years of age [2]. Notably, many older adults with severe illness require hospital admission. For example, in the Canadian Serious Outcomes Surveillance (SOS) Network of acute care hospitals, 3394 adults were admitted to hospital with laboratory-confirmed influenza over three consecutive influenza seasons (2011/2012 through 2013/2014), of whom 2078 (61.2\%) were aged 65 years and over. At $9.1 \%$, mortality was high in the overall cohort, and increased with age $3.5 \%$ for ages $16-49$ years, $6.2 \%$ for ages $50-64$ years, $6.9 \%$ for ages $65-75$ years, and $14.3 \%$ for those aged $>75$ years) [3]. Experience from other national and international surveillance networks paints a similar picture of serious outcomes being more often experienced by older adults; this evidence has contributed to international advisory body recommendations as discussed below. Despite the severity of outcomes associated with influenza, and recommendations for vaccination for older adults and other high-risk groups in many jurisdictions, vaccine coverage remains below the $75-80 \%$ target for the over 65 years population [4]. For example, in the United States [5] and Canada [6], vaccination rates among older adults have plateaued and remain below 70\%. In the European Union, influenza vaccine coverage in high-risk groups has declined over the period from 2007 to 2015 ; for those aged $65+$ years, coverage varied widely $(1.0-78.7 \%)$ across member states, with a median vaccination rate of $47.6 \%$ [7]. In Australia, 2012-2013 estimates of vaccine coverage were 70.9 and $64.4 \%$ for Australian-born and immigrant older adults aged $65+$ years, respectively [8]. Vaccine coverage in some populations may be higher; a 2013 study by the Australian Influenza Complications Alert Network estimated influenza vaccine coverage at $81 \%$ for test-negative control inpatients aged 65+years [9], although it is difficult to extrapolate hospital-based estimates to the general population.

It is becoming increasingly evident that longer-term complications are also common. Following an acute care hospitalization, a substantial proportion of older adults will not return to their prior baseline of health and functional status [10]. In the SOS Network, an estimated 19\% of older adults aged $65+$ years admitted with respiratory illness, including laboratory-confirmed influenza, suffered catastrophic disability (defined as persistent loss of function in two or more activities of daily living 30 days following hospital discharge) [11, 12]. Even older adults who are not hospitalized can experience persistent declines in function and prolonged recovery from influenza or influenza-like illness. A Canadian survey of 5014 relatively fit older adults found that $39.3 \%$ of those reporting having experienced influenza or an influenza-like illness during the most recent influenza season took longer than 2 weeks to recover, one-fifth reported needing new assistance in daily functional tasks, $13.9 \%$ were admitted to hospital, and $3.1 \%$ never fully recovered [13].

Frailty is an important concept when it comes to older adults and influenza, and can be measured and defined in many ways. Generally speaking, frailty represents vulnerability to adverse outcomes [14]. Frailty is associated with reduced vaccine effectiveness, and is therefore important to consider in studies of vaccine protection [15]. Frailty also predicts outcomes from influenza illness; frail older adults are more likely to suffer adverse outcomes and less likely to return to their prior baseline function $[11,12]$. As such, frail older adults have the most to lose from an influenza infection and the most to gain in preventing it, but have poorer responses to vaccination. This makes improved vaccination strategies, along with other preventive approaches, all the more important for this vulnerable population.

It is important to distinguish between vaccine efficacy and vaccine effectiveness. Vaccine efficacy estimates are derived from research study settings, usually randomized controlled studies, under ideal conditions. In contrast, vaccine effectiveness reflects the benefit seen with a particular vaccine in real-world settings [16]. Confusingly, both can be abbreviated as 'VE', therefore it is important to know to which a particular study is referring. It is also important to clearly define the outcomes measured in vaccine-effectiveness studies. Many studies, for example from outpatient sentinel surveillance networks, report vaccine effectiveness (VE) for the prevention of medically attended influenza [17-19]. This is an important outcome, although it generally captures and reflects milder cases that do not lead to hospital admission. Certain age groups (for example, working-age adults who require sick notes for missed work) are generally overrepresented in outpatient sentinel surveillance, while others (for example, older adults, particularly those who are frail) are underrepresented. These studies are important for describing the circulation of influenza strains over geographical areas, and, because they capture illness that leads to missed work or school, are also critical in informing economic 
evaluations of the impact of influenza [20-23]. Other studies report VE in the prevention of serious outcomes, such as hospital admissions, intensive care unit admissions, need for mechanical ventilation, and death [3, 15, 24-26]. Vaccineeffectiveness estimates, even for the same product and in the same influenza season and strain circulation, may therefore be different between studies that report on the prevention of medically attended influenza and those that examine prevention of severe outcomes [27]. For example, a report from the European I-MOVE network study in the 2016/2017 influenza season found an adjusted VE against medically attended influenza in primary care of $38.0 \%$ (95\% confidence interval [CI] 21.3-51.2) for all age groups; among adults, the VE was lower in those $\geq 65$ years of age $(23.4 \%$; $95 \%$ CI -15.4 to 49.1$)$ than in those aged $15-64$ years $(46.9 \%, 95 \%$ CI 25.2-62.3). The same study also reported much lower VE against more severe illness requiring hospital admission in that season; the adjusted VE was $2.5 \%$ (95\% CI -43.6 to 33.8) for those aged $\geq 65$ years [27].

\subsection{Vaccine Effectiveness of Flu Vaccine Traditional Products}

Traditional influenza vaccines are either split-virus or subunit vaccines that contain distinct antigens that are selected to match predicted circulating strains each season, and standardized according to the content of hemagglutinin (HA; standard dose $=15 \mathrm{ug}$ ) for each of the vaccine strains. The hemagglutination inhibition (HAI) assay is the gold-standard assay of antibody titers against HA and is used as a surrogate of vaccine efficacy for the prevention of influenza infection. In addition to HA, subunit and split-virus vaccines contain unspecified quantities of neuraminidase (NA). Splitvirus vaccines also contain internal proteins of the virus, most notably matrix (M1) protein and nucleoprotein (NP). Trivalent vaccines contain three strain antigens (two influenza A strains, A/H1 and A/H3) and one B lineage (either Victoria or Yamagata). Since it is difficult to predict which of the B lineages will be circulating in a given year (and indeed, they sometimes co-circulate) [25], quadrivalent vaccines have been developed to include both B lineages. Strain selection is done separately for the Northern and Southern Hemisphere products.

While antibody responses play a role in the prevention of influenza infection, cell-mediated immune responses play a role in both the prevention of infection and of serious complications of influenza, especially when antibody levels are low (as in the case of an influenza pandemic), or fail to prevent infection (as is often the case in older adults) [28]. Compared with the usual surface protein vaccine targets (HA and NA), internal virus proteins evolve more slowly and are more often preserved across influenza, strains, types and subtypes, making them attractive potential vaccine targets. Cell-mediated immunity, notably cytotoxic T lymphocyte (CTL) responses to internal viral proteins, including M1 and $\mathrm{NP}$, is increasingly recognized as important in protecting against severe outcomes of influenza; CTLs are required to clear influenza from the lungs. Moreover, in contrast to the strain-specific antibody response to HA and NA, the CTL response to influenza A (or B) is cross-protective because the internal viral proteins (including M1 and NP) are shared across all influenza strains, and immunologic memory can be recalled from prior exposure to influenza A (or B) through infection or vaccination.

Many factors contribute to fluctuations in VE estimates across seasons, including differences in strain circulation and epidemiology, drift and mismatch with vaccine strains, and historical cohort immunity (or lack thereof). As such, it is difficult to generalize VE estimates across seasons and between jurisdictions. In general, influenza VE tends to be moderate, but, again, season, strain and subgroup differences abound. A recent meta-analysis of 30 studies from the 2010/2011 through 2014/2015 seasons found a pooled VE of $41 \%$ (95\% CI 34-48) for the prevention of hospital admission from influenza, and VE was lower among older adults aged $65+$ years, at 37\% (95\% CI 30-44), versus younger adults aged 18-64 years, at 51\% (95\% CI 44-58) [29]. In pooled results from the Canadian SOS Network, across three influenza seasons (2011/2012-2013/2014), VE was 48.0\% (95\% CI 37.5-56.7) for adults 19-64 years of age, and 39.3\% (95\% CI 29.4-47.8) in those 65 + years of age. Notably, VE was higher for the prevention of the most serious outcomes; VE for the prevention of any influenza-associated death was $74.5 \%$ (95\% CI 44.0-88.4) for those aged 65 + years [3].

Crucially, although VE is usually thought to be lower in older adults than in younger adults, actually this is not always the case. In reports from the 2011/2012 season in the Canadian SOS Network, adjusted VE for preventing hospitalization was $42.8 \%$ (95\% CI 23.8-57.0) for all adults aged $\geq 16$ years, but was lower for adults aged 16-64 years (33.2\%; 95\% CI -6.7 to 58.2) than for those aged $\geq 65$ years (58.0\%; 95\% CI 34.2-73.2) [15, 25]. Clearly, seasonal differences in circulating strains will play an important role (e.g. when a type or subtype predominates, i.e. A(H3N2), A(H1N1)pdm, B Victoria, B Yamagata, or some combination of these; interestingly, during the 2011/2012 season in Canada, all four of these strains co-circulated). The greatest impact in older adults occurs during years when $\mathrm{A} / \mathrm{H} 3 \mathrm{~N} 2$ is the predominant circulating strain. Lower hospitalization rates of older adults during years when $\mathrm{pH} 1 \mathrm{~N} 1$ predominates may be explained by 'immunologic memory' from childhood exposure to similar H1N1 strains conferring protection in the current cohort of older adults. Other potential contributors to these findings include differences in control group composition (e.g. if the younger adults admitted to hospital 
tended to have more comorbidities that would also affect the response to influenza vaccination), and robust accounting for confounders, including frailty [30].

Regardless of the details of differences in VE across seasons and age groups, it is clear that older adults are disproportionately affected by influenza and its complications, and better vaccines are an important part of addressing this problem.

\section{Influenza and the Aging Immune System}

Functional integrity of the immune system is affected by aging, manifesting as reductions in humoral immunity (with declines in antibody titers and decreased antibody avidity), reductions in certain aspects of cell-mediated immunity, and dysregulation of cytokine responses needed to activate both innate and adaptive immune mechanisms. For example, anti-inflammatory responses protect against the tissuedamaging effects of chronic inflammation associated with many chronic diseases, referred to as 'inflammaging' [31]. However, in the setting of acute infection such as influenza, regulation of these inflammatory processes is needed to turn on cell-mediated immune mechanisms and protect against tissue damage. Changes in antigen processing and presentation may also be involved. Aging is also associated with weakening host defenses such as mucociliary clearance and less effective cough (e.g. stemming from sarcopenia or reductions in muscle strength) [32].

Given these changes across all facets of the immune system, traditional measures of vaccine response such as antibody titers do not correlate well with strain-specific vaccine efficacy. This presents challenges for predicting responses to vaccination. Antibody responses are routinely used to screen new vaccines but their limitations as sole predictors of vaccine efficacy are increasingly recognized [33-36]. For instance, vaccinated older adults who develop laboratoryconfirmed influenza illness due to $\mathrm{A} / \mathrm{H} 3 \mathrm{~N} 2$ infection have similar A/H3N2-specific antibody titers following vaccination compared with those who do not develop laboratoryconfirmed influenza [37-39], suggesting that antibody titers alone cannot predict strain-specific vaccine efficacy.

Across the lifespan, responses of the immune system are the result of interactions between innate versus adaptive immunity and associated regulatory pathways. Innate immunity consists of non-clonotypic responses to pathogen challenge, including physical and chemical barriers, phagocytic cells, natural killer cells and plasma proteins. Notably, features that are beneficial early in life (e.g. a robust response to novel infections, supporting early-life survival), may contribute to inflammatory illness later in life if not kept in check by anti-inflammatory regulatory processes [31]. The latter rely inter alia on CD4+ T cells (including
T-helper [Th] cells and regulatory T cells). The subsets of Th lymphocytes are ideally in balance; Th1 are generally pro-inflammatory, while Th2 are anti-inflammatory [40]. Regulatory pathways involving different types of CD4+ T cells are crucial for maintaining an appropriate balance between fighting off threats (pathogens), not attacking self (auto-immunity), and preventing chronic inflammation on resolution of pathogen challenge.

Pandemic influenza A/H1N1 (pH1N1) provides an interesting example of the critical roles of both humoral and cellmediated immunity. It has been suggested that older adults aged $65+$ years have pre-existing memory from early childhood exposure to $\mathrm{pH} 1 \mathrm{~N} 1$-related strains that is re-stimulated with vaccination, leading to lower attack rates when $\mathrm{pH} 1 \mathrm{~N} 1$ strains circulate [41]. However, in the very old ( $>80$ years of age), higher $\mathrm{pH} 1 \mathrm{~N} 1$ antibody titers relative to 65 - to 79-yearolds do not necessarily translate to lower influenza illness rates [42], and diminished cell-mediated immunity with increasing age may contribute to greater severity once older people become infected [41]. Therefore, although humoral memory provides some protection against the initial steps of infection, once the infection takes hold the aging immune system has a harder time fighting it off.

Vaccination brings important opportunities to hone and prepare immune responses, even in older adults. Prior exposure to influenza through infection or vaccination has a greater impact on antibody titers and antibody responses to vaccination than aging, which may not be so deleterious as previously believed. In contrast, the decline in cell-mediated immune responses to influenza is related to aging, rather than exposure to the virus, and may lead to age-associated increased susceptibility to disease [41]. Age-related differences in $\mathrm{T}$ cell responses have been associated with a decline in the antibody response to influenza vaccination [43, 44], but, in contrast to cellular immunity, this appears to be more related to the effect of annual repeated vaccination rather than age per se [45]. Notably, inducible activity levels of cytolytic mediators, Granzyme B (GrB), and the ratio of interferon (IFN)- $\gamma$ (pro-inflammatory) to interleukin (IL)-10 (anti-inflammatory) [IFN $\gamma$ :IL-10 ratio] secreted by peripheral blood mononuclear cells (PBMCs) challenged ex vivo with live flu virus predict a protective response to $\mathrm{A} / \mathrm{H} 3 \mathrm{~N} 2$ infection; older adults who go on to develop influenza illness have low IFN $\gamma$ :IL-10 ratios and low Ievels of inducible GrB activity $[37,46]$. These low levels are also highly correlated with influenza illness severity [38]. Additionally, in these vaccine 'non-responders', influenza A/H3N2 infection fully restored the GrB response to influenza challenge to that of uninfected individuals in the study cohort [38]. Those 'non-responders' who have had a recent influenza illness mount an even greater GrB response to a subsequent influenza vaccination [37]. The fact that influenza infection can stimulate the immune response in older adults in ways 
that vaccines currently cannot reproduce suggests that weak cell-mediated immune responses to influenza vaccination are a limitation of the vaccine rather than of the aging immune system. Hence, efforts at improving influenza vaccines for older adults should focus on increasing the potency of cellmediated immunity.

Options that have been explored to date for improved influenza vaccines include increasing the dose of antigen to better stimulate weaker adaptive responses, adding chemical agents (adjuvants) that stimulate the innate inflammatory response and enhance the ability of dendritic cells to present antigen to $\mathrm{T}$ cells, thus bringing more exposure to adaptive immune cells in the reactive milieu which ensues, and using recombinant antigens.

\section{Overview of Currently Available Influenza Vaccine Products Specifically Targeted to Older Adults}

\subsection{High-Dose Influenza Vaccine}

To enhance the antibody response in older individuals, high-dose antigen vaccines have been developed in an effort to increase vaccine efficacy/effectiveness. As shown in Table 1, the current high-dose formulation is a split-virus influenza vaccine (includes M1 and NP) containing four times the dose of each of the three influenza antigens in comparison with standard-dose trivalent vaccines. Multiple studies demonstrate that this strategy elicits a more robust immune response in older individuals, even those with multiple comorbidities and those who are frail. This enhanced immune response appears to translate into improved vaccine efficacy. In a large phase IIIb/IV study of 31,989 subjects aged 65 years or older over two influenza seasons, highdose trivalent influenza vaccine (TIV) demonstrated better protection against laboratory-confirmed influenza over the standard-dose trivalent vaccine (relative efficacy $24.1 \%, 95 \%$ CI 9.7-36.5). Importantly, this benefit was observed even in those older than 75 years of age (relative efficacy $32.4 \%$, 95\% CI 12.5-52.5) and among participants with laboratoryconfirmed influenza $\mathrm{A}(\mathrm{H} 3 \mathrm{~N} 2)$ [relative efficacy $23.3 \%$,
95\% CI 6.0-37.5] [47]. These findings are supported by a meta-analysis of seven trials, which found a significantly reduced risk of developing laboratory-confirmed influenza among older persons receiving high-dose vaccine versus those having received standard dose (risk ratio $0.76,95 \%$ CI 0.65-0.90) [48]. In a study conducted as a collaboration between the US FDA, the Centers for Disease Control and Prevention, and the Centers for Medicare and Medicaid Services (CMS), analysis of the CMS database for the 2012/2013 influenza season among approximately 2.5 million Medicare beneficiaries reported high-dose TIV was found to have an overall relative effectiveness of $22 \%$ (95\% CI 15-29) for the prevention of laboratory-confirmed influenza, and relative effectiveness of $22 \%$ (95\% CI 16-27) for the prevention of influenza-related emergency room visits or hospitalizations. Among persons 85 years and older, highdose TIV was 36\% (95\% CI 13-54) more effective in the prevention of laboratory-confirmed influenza [49]. Benefit of the high-dose TIV compared with standard TIV appears to be greatest against influenza $\mathrm{A}(\mathrm{H} 3 \mathrm{~N} 2)$. In a comparative effectiveness study among US Medicare beneficiaries, overall relative effectiveness of high-dose TIV was $24 \%$ (95\% CI 0.6-42) for the prevention of 30-day mortality following an emergency room visit or hospitalization with an administrative code for influenza over two influenza seasons; relative effectiveness was $36.4 \%$ (95\% CI 9-56) during the 2012/2013 season during which influenza A(H3N2) circulation dominated, and $2.5 \%$ (95\% CI - 47 to 35$)$ during the 2013/2014 season dominated by circulation of influenza A(H1N1) [50]. A more recent, large observational study of over 200,000 veterans aged 65 years and older demonstrated a relative vaccine efficacy of $25 \%$ (95\% CI 2-43) against influenza- or pneumonia-associated hospitalization for highdose versus standard-dose recipients [51].

A single-blind, cluster-randomized trial of US nursing home residents demonstrated a significant reduction in risk of hospital admission for respiratory illness during a single influenza season among facilities that used high-dose vaccine versus those that administered standard dose (RR 0.873 , 95\% CI 0.776-0.9882) [52].

Despite its higher product cost, high-dose vaccine has been found to be cost effective due to a reduction in overall

Table 1 Influenza vaccine formulations available for older adults

\begin{tabular}{|c|c|c|c|c|}
\hline Vaccine & Type & Content & Dose, $\mathrm{mL}$ & Route \\
\hline Inactivated tri- or quadrivalent vaccine & Subunit & 15 ug HA per antigen & 0.5 & IM \\
\hline Adjuvanted inactivated trivalent influenza vaccine & Subunit & $\begin{array}{l}\text { MF59 adjuvant } \\
15 \text { ug HA per antigen }\end{array}$ & 0.5 & IM \\
\hline High-dose inactivated trivalent influenza vaccine & Subunit & 60 ug HA per antigen & 0.5 & IM \\
\hline Recombinant quadrivalent influenza vaccine & Recombinant & 45 ug rHA per antigen & 0.5 & IM \\
\hline
\end{tabular}

$H A$ hemagglutinin, $r H A$ recombinant hemagglutinin, $I M$ intramuscularly 
influenza-related medical encounters, particularly hospitalizations [53,54]. Based on an economic analysis of the phase IIIb/IV study, using the healthcare payer perspective, highdose vaccine was indeed shown to be cost saving compared with standard-dose vaccine. Overall costs (US\$) associated with standard-dose vaccine were $\$ 116$ higher for all participants, $\$ 106$ higher for participants with at least one comorbid disorder, and $\$ 12$ higher for participants aged 75 years and older. Cost differences were slightly higher when estimated using a societal perspective $(\$ 128, \$ 119$, and $\$ 22$ for all participants, those with comorbid disorders, and those aged 75 years or older, respectively) [54].

Taken together, these data suggest that high-dose influenza vaccine reduces laboratory-confirmed influenza and influenza-related hospitalizations even among nursing home residents, who are more likely to be frail, and is cost effective when compared with standard-dose influenza vaccine. This evidence base has led some jurisdictions to provide preferential wording in their recommendations regarding high-dose vaccine in older adults (see section on Advisory Body Recommendations).

\subsection{Adjuvanted Influenza Vaccine}

Adjuvants have been added to subunit vaccine formulation to enhance antibody response to vaccination. Adjuvanted TIV contains MF59, an oil-in-water emulsion of squalene, which potentiates immune response by recruiting and activating immune cells at the injection site [44]. This in turn allows for greater uptake, transportation and processing of the antigens, allowing for improved T-cell priming $[44,55]$. Numerous studies have shown that older adults, including those with comorbidities, as well as those residing in nursing home settings, exhibit a greater immune response to MF59-adjuvanted influenza vaccine in comparison with non-adjuvanted formulations [56-58].

While there are no randomized controlled trials directly comparing vaccine efficacy between MF59 adjuvanted and non-adjuvanted formulations in older persons, a recent meta-analysis pooled VE data from several observational studies [59]. Adjuvanted vaccine was more effective than non-adjuvanted vaccine in preventing laboratory-confirmed influenza (odds ratio [OR] 0.37, 95\% CI 0.14-0.96) and in preventing hospitalizations due to pneumonia/influenza (RR $0.75,95 \%$ CI $0.57-0.98)$. Furthermore, results from the single study conducted in a long-term care setting found a VE of adjuvanted vaccine of $94 \%$ (95\% CI 47-100) in reducing influenza-like illness among older residents of long-term care facilities. Those with underlying chronic cardiorespiratory diseases demonstrated the greatest benefit [60]. VE was 51\% (95\% CI 39-61) for preventing hospitalization secondary to pneumonia/influenza in older community-dwelling adults. Interestingly, adjuvanted vaccine was also effective in reducing admissions for both acute coronary syndrome and cerebrovascular disease (VE 87\%, 95\% CI 35-97, and VE 93\%, 95\% CI 52-99, respectively) [61]. Overall, these data suggest that MF59-adjuvanted influenza vaccine may be associated with a reduced risk of influenza-related complications in older adults in comparison with standard-dose influenza vaccine; this is reflected in some advisory statements (see section on Advisory Body Recommendations).

\subsection{Recombinant Influenza Vaccine}

Recombinant influenza vaccine (RIV) utilizes DNA recombinant technology to produce influenza HA protein in cell culture rather than cultivating live influenza virus in embryonated hen eggs [62]. A number of RIV formulations are in various stages of development and marketing.

While originally indicated for adults 18-49 years of age, an RIV vaccine made using a baculovirus/insect cell system has recently been approved by the US FDA for adults aged 50 years and older [63]. In addition to utilizing unique technology, this vaccine contains three times the HA of the standard influenza vaccine (Table 1). Consequently, there is growing interest regarding the efficacy of RIV in older persons. Although no studies have specifically compared recombinant vaccine with standard-dose vaccine in those aged 65 years and older, one clinical trial included a subgroup analysis of this population [64]. When compared with inactivated, quadrivalent influenza vaccine (QIV), the quadrivalent RIV containing recombinant HA proteins demonstrated a relative vaccine efficacy of $42 \%(95 \%$ CI 9-65) in the prevention of culture-positive, protocol-defined, influenza-like illness among participants aged $65+$ years. Further research is needed to determine if this and other RIVs reduce the risk of influenza and its complications in older individuals.

\section{Advisory Body Recommendations Regarding Seasonal Influenza Vaccination for Older Adults}

Many advisory bodies recommend seasonal influenza vaccination for older adults due to their high risk of influenza illness and its complications [4, 63, 65-67]. The Canadian National Advisory Committee on Immunization (NACI) statement mentions standard-dose TIV and QIV, adjuvanted TIV, and high-dose TIV as approved options for older adults, and reads: "Based on the available evidence, NACI concludes that there is evidence that high dose TIV should provide superior protection compared with standard-dose TIV for adults $\geq 65$ years of age. This superior relative protection compared to standard-dose TIV appears to increase with increasing age over 65 years" [67]. The United States 
Advisory Committee on Immunization Practices (ACIP) lists trivalent and quadrivalent, standard- or high-dose, adjuvanted or unadjuvanted, or RIVs as potential options for older adults, and states that high-dose influenza vaccine may provide better protection than standard-dose vaccine for this age group [63]. The Australian Immunization Handbook states that high-dose and adjuvanted influenza vaccines are both preferentially recommended compared with other available influenza vaccines for older adults aged 65 years and older; no preference is expressed between the two [65].

On the other hand, other advisory bodies have not made or suggested potential prioritization recommendations, including the European Centre for Disease Prevention and Control (ECDC) and the World Health Organization $[4,66]$. The ECDC mentions that QIVs are available in some European countries and they anticipate that they will replace TIV over time, although they do not specifically mention their use in older adults. The ECDC also mentions adjuvanted vaccines, which are available in some but not all European jurisdictions, stating: "It is currently not clear if any of them perform better than the unadjuvanted vaccines although the first studies indicate better protection in the oldest age group" [68]. High-dose vaccines and RIVs are not mentioned in the 2017-2018 ECDC guidelines. Recombinant vaccines are at a relatively early stage of evidence, and this is reflected in the lack of specific advisory statements about the use of RIV in older adults, other than the ACIP mention of RIV as an available option in this population [63]. As the newer vaccine products are introduced, they tend to have relatively low coverage. This means that the availability of postmarketing 'real world' evidence is limited as the field aims to advance the results of randomized controlled trials and inform policy and inform advisory statement recommendations.

As emphasized in the ACIP published recommendations paper, no comparative data are available between the newer products, which they conclude prevents recommending one over another in older adults. Indeed, based on the pressing need to improve vaccine coverage, they emphasize that "vaccination should not be delayed if a specific product is not readily available" [63].

\section{Conclusions}

Older adults are vulnerable to poor outcomes from influenza over both short- and long-term time horizons. Both complications of acute illness and persistent functional disability have important impacts on the health and well-being of older adults and their loved ones. Prevention is therefore of upmost importance as a strategy to support healthy aging. Even though immune responses to vaccination may be suboptimal in older adults, particularly those who are frail, vaccination continues to be an important tool in the prevention of severe outcomes from influenza. Several vaccine products are available for older adults, including standard-dose trivalent and quadrivalent formulations of split virus and subunit vaccines, high-dose split-virus vaccine, adjuvanted subunit vaccine, and recombinant $\mathrm{HA}$ vaccine. Because the relative merits and availability of these products may vary between jurisdictions, vaccinating with whatever appropriate and approved product is available remains a prime recommendation; vaccination coverage remains suboptimal in most jurisdictions. As our understanding of immune changes with aging, and frailty progresses, vaccine products will ideally be further tailored to generate optimal protection for this vulnerable population.

\section{Compliance with Ethical Standards}

Conflict of interest Melissa K. Andrew reports grant funding from GlaxoSmithKline (GSK), Pfizer, Sanofi, Canadian Institute of Health Research (CIHR), Public Health Agency of Canada (PHAC), and the Canadian Frailty Network; no personal financial conflicts of interest. Susan K. Bowles reports grant funding from the Dalhousie Pharmacy Endowment Fund; no financial conflicts of interest. Graham Pawelec reports no financial conflicts of interest. Laura Haynes reports grant funding from the National Institutes of Health (NIH; AG021600); no financial conflicts of interest. George A. Kuchel reports grant funding from the NIH (AG048023, AG021600, AI124297, AG052608, GM124922, AG056925), Patient-Centered Outcomes Research Institute (PCORI) and Novartis; no personal financial conflicts of interest. He is also supported by the Travelers Chair in Geriatrics and Gerontology. Shelly A. McNeil reports grants and payments from the GSK group of companies, Pfizer, Merck, Novartis, Sanofi, PHAC and CIHR. Janet E. McElhaney reports payments to her institution from GSK, Sanofi, and Pfizer, and is supported by the Health Sciences North Volunteer Association Research Chair in Healthy Aging.

Funding None of the authors received any funding or payment for the preparation of this review.

\section{References}

1. Nichol KL. Influenza vaccination in the elderly: impact on hospitalisation and mortality. Drugs Aging. 2005;22(6):495-515.

2. Iuliano AD, Roguski KM, Chang HH, Muscatello DJ, Palekar R, Tempia S, et al. Estimates of global seasonal influenzaassociated respiratory mortality: a modelling study. Lancet. 2018;391(10127):1285-300.

3. Nichols MK, Andrew MK, Hatchette TF, Ambrose A, Boivin G, Bowie W, et al. Influenza vaccine effectiveness to prevent influenza-related hospitalizations and serious outcomes in Canadian adults over the 2011/12 through 2013/14 influenza seasons: a pooled analysis from the Canadian Immunization Research Network (CIRN) Serious Outcomes Surveillance (SOS Network). Vaccine. 2018;36(16):2166-75.

4. World Health Organization: Seasonal Influenza Fact Sheet 2018. http://www.who.int/mediacentre/factsheets/fs211/en/. Accessed 24 Oct 2018.

5. Centers for Disease Control and Prevention. Flu Vaccination Coverage, United States, 2016-17 Influenza Season. https://www.cdc.gov/ flu/fluvaxview/coverage-1617estimates.htm. Accessed 24 Oct 2018. 
6. Public Health Agency of Canada. 2016/17 seasonal influenza vaccine coverage in Canada. 2018. http://publications.gc.ca/collection s/collection_2018/aspc-phac/HP40-198-2017-eng.pdf. Accessed 24 Oct 2018

7. European Cenre for Disease Prevention and Control. Seasonal influenza vaccination in Europe-vaccination recommendations and coverage rates for eight influenza seasons (2007-2008 to 2014-2015). 2017. https://ecdc.europa.eu/en/publicationsdata/seasonal-influenza-vaccination-europe-vaccination-recom mendations-and-coverage-2007-2015. Accessed 24 Oct 2018.

8. Karki S, Dyda A, Newall A, Heywood A, MacIntyre CR, McIntyre $\mathrm{P}$, et al. Comparison of influenza vaccination coverage between immigrant and Australian-born adults. Vaccine. 2016;34(50):6388-95.

9. Cheng AC, Dwyer DE, Holmes M, Irving LB, Brown SG, Waterer GW, et al. Influenza epidemiology, vaccine coverage and vaccine effectiveness in sentinel Australian hospitals in 2013: the influenza complications alert network. Commun Dis Intell Q Rep. 2014;38(2):E143-9.

10. Covinsky KE, Palmer RM, Fortinsky RH, Counsell SR, Stewart AL, Kresevic D, et al. Loss of independence in activities of daily living in older adults hospitalized with medical illnesses: increased vulnerability with age. J Am Geriatr Soc. 2003;51(4):451-8.

11. Andrew MK, Lees C, Godin J, Black K, McElhaney J, et al. Frailty hinders recovery from acute respiratory illness in older adults [abstract]. In: ID Week: 4-8 Oct 2017; San Diego, CA.

12. Andrew MK, MacDonald S, Ye L, Ambrose A, et al., on behalf of the CIRN SOS Network Investigators and the TIBDN Investigators. Impact of frailty on influenza vaccine effectiveness and clinical outcomes: experience from the Canadian immunization research network (CIRN) Serious Outcomes Surveillance (SOS) Network 2011/12 Season [abstract]. In: 12th Canadian Immunization Conference, Ottawa, ON, 6-8 Dec 2016.

13. Andrew MK, Gilca V, Waite N, Pereira JA. Examining the knowledge, attitudes and experiences of canadian seniors towards influenza (the EXACT survey) [abstract]. In: Canadian immunization conference, Ottawa, ON, 4-6 Dec 2018.

14. Clegg A, Young J, Iliffe S, Rikkert MO, Rockwood K. Frailty in elderly people. Lancet. 2013;381(9868):752-62.

15. Andrew MK, Shinde V, Ye L, Hatchette T, Haguinet F, Dos Santos G, et al. The importance of frailty in the assessment of influenza vaccine effectiveness against influenza-related hospitalization in elderly people. J Infect Dis. 2017;216(4):405-14.

16. Weinberg GA, Szilagyi PG. Vaccine epidemiology: efficacy, effectiveness, and the translational research roadmap. J Infect Dis. 2010;201(11):1607-10.

17. van Doorn E, Darvishian M, Dijkstra F, Donker GA, Overduin $\mathrm{P}$, Meijer A, et al. Influenza vaccine effectiveness estimates in the Dutch population from 2003 to 2014: the test-negative design case-control study with different control groups. Vaccine. 2017;35(21):2831-9.

18. Cowling BJ, Feng S, Finelli L, Steffens A, Fowlkes A. Assessment of influenza vaccine effectiveness in a sentinel surveillance network 2010-13, United States. Vaccine. 2016;34(1):61-6.

19. Skowronski DM, Chambers C, De Serres G, Sabaiduc S, Winter AL, Dickinson JA, et al. Age-related differences in influenza b infection by lineage in a community-based sentinel system, 20102011 to 2015-2016, Canada. J Infect Dis. 2017;216(6):697-702.

20. Cohen JM, Silva ML, Caini S, Ciblak M, Mosnier A, Daviaud I, et al. Striking similarities in the presentation and duration of illness of influenza A and B in the community: a study based on sentinel surveillance networks in France and Turkey, 20102012. PLoS One. 2015;10(10):e0139431.

21. Gianino MM, Politano G, Scarmozzino A, Charrier L, Testa M, Giacomelli S, et al. Estimation of sickness absenteeism among
Italian healthcare workers during seasonal influenza epidemics. PLoS One. 2017;12(8):e0182510.

22. Jackson ML, Phillips CH, Benoit J, Jackson LA, Gaglani $\mathrm{M}$, Murthy $\mathrm{K}$, et al. Burden of medically attended influenza infection and cases averted by vaccination-United States, 2013/14 through 2015/16 influenza seasons. Vaccine. 2018;36(4):467-72.

23. Van Wormer JJ, King JP, Gajewski A, McLean HQ, Belongia EA. Influenza and workplace productivity loss in working adults. J Occup Environ Med. 2017;59(12):1135-9.

24. El Omeiri N, Azziz-Baumgartner E, Thompson MG, REVELAC-i network participants, Clara W, Cerpa M, et al. Seasonal influenza vaccine effectiveness against laboratory-confirmed influenza hospitalizations - Latin America, 2013. Vaccine. 2018;36(24):3555-66.

25. Andrew MK, Shinde V, Hatchette T, Ambrose A, Boivin G, Bowie $\mathrm{W}$, et al. Influenza vaccine effectiveness against influenza-related hospitalization during a season with mixed outbreaks of four influenza viruses: a test-negative case-control study in adults in Canada. BMC Infect Dis. 2017;17(1):805.

26. Petrie JG, Ohmit SE, Cheng CK, Martin ET, Malosh RE, Lauring AS, et al. Influenza vaccine effectiveness against antigenically drifted influenza higher than expected in hospitalized adults: 2014-2015. Clin Infect Dis. 2016;63(8):1017-25.

27. Kissling E, Rondy M, I-MOVE/I-MOVE+ study team. Early 2016/17 vaccine effectiveness estimates against influenza A(H3N2): I-MOVE multicentre case control studies at primary care and hospital levels in Europe. Eur Surveill. 2017. https:// doi.org/10.2807/1560-7917.ES.2017.22.7.30464.

28. Sridhar S, Begom S, Bermingham A, Hoschler K, Adamson W, Carman W, et al. Cellular immune correlates of protection against symptomatic pandemic influenza. Nat Med. 2013;19(10):1305-12.

29. Rondy M, El Omeiri N, Thompson MG, Leveque A, Moren A, Sullivan SG. Effectiveness of influenza vaccines in preventing severe influenza illness among adults: a systematic review and meta-analysis of test-negative design case-control studies. J Infect. 2017;75(5):381-94.

30. Falsey AR, McElhaney JE. Influenza burden in frail elderly. Lancet Respir Med. 2018;6(1):e2.

31. Franceschi C, Capri M, Monti D, Giunta S, Olivieri F, Sevini F, et al. Inflammaging and anti-inflammaging: a systemic perspective on aging and longevity emerged from studies in humans. Mech Ageing Dev. 2007;128(1):92-105.

32. Larbi A, Franceschi C, Mazzatti D, Solana R, Wikby A, Pawelec G. Aging of the immune system as a prognostic factor for human longevity. Physiology (Bethesda). 2008;23:64-74.

33. Murasko DM, Bernstein ED, Gardner EM, Gross P, Munk G, Dran $\mathrm{S}$, et al. Role of humoral and cell-mediated immunity in protection from influenza disease after immunization of healthy elderly. Exp Gerontol. 2002;37(2-3):427-39.

34. Effros RB. Role of T lymphocyte replicative senescence in vaccine efficacy. Vaccine. 2007;25(4):599-604.

35. McElhaney JE, Effros RB. Immunosenescence: what does it mean to health outcomes in older adults? Curr Opin Immunol. 2009;21(4):418-24.

36. McElhaney JE. Prevention of infectious diseases in older adults through immunization: the challenge of the senescent immune response. Expert Rev Vaccines. 2009;8(5):593-606.

37. McElhaney JE, Ewen C, Zhou X, Kane KP, Xie D, Hager WD, et al. Granzyme B: correlates with protection and enhanced CTL response to influenza vaccination in older adults. Vaccine. 2009;27(18):2418-25.

38. Shahid Z, Kleppinger A, Gentleman B, Falsey AR, McElhaney JE. Clinical and immunologic predictors of influenza illness among vaccinated older adults. Vaccine. 2010;28(38):6145-51. 
39. Merani S, Kuchel GA, Kleppinger A, McElhaney JE. Influenza vaccine-mediated protection in older adults: impact of influenza infection, cytomegalovirus serostatus and vaccine dosage. Exp Gerontol. 2018;107:116-25.

40. Berger A. Th1 and Th2 responses: what are they? BMJ. 2000;321(7258):424.

41. Skowronski DM, Hottes TS, McElhaney JE, Janjua NZ, Sabaiduc $\mathrm{S}$, Chan T, et al. Immuno-epidemiologic correlates of pandemic H1N1 surveillance observations: higher antibody and lower cellmediated immune responses with advanced age. J Infect Dis. 2011;203(2):158-67.

42. Skowronski DM, Chambers C, Sabaiduc S, Janjua NZ, Li G, Petric M, et al. Pre- and postpandemic estimates of 2009 pandemic influenza $\mathrm{A}(\mathrm{H} 1 \mathrm{~N} 1)$ seroprotection to inform surveillance-based incidence, by age, during the 2013-2014 epidemic in Canada. J Infect Dis. 2015;211(1):109-14.

43. Goronzy JJ, Fulbright JW, Crowson CS, Poland GA, O'Fallon WM, Weyand CM. Value of immunological markers in predicting responsiveness to influenza vaccination in elderly individuals. J Virol. 2001;75(24):12182-7.

44. Saurwein-Teissl M, Lung TL, Marx F, Gschosser C, Asch E, Blasko I, et al. Lack of antibody production following immunization in old age: association with CD8(+)CD28(-) T cell clonal expansions and an imbalance in the production of Th1 and Th2 cytokines. J Immunol. 2002;168(11):5893-9.

45. Mosterin Hopping A, McElhaney J, Fonville JM, Powers DC, Beyer WEP, Smith DJ. The confounded effects of age and exposure history in response to influenza vaccination. Vaccine. 2016;34(4):540-6.

46. McElhaney JE, Xie D, Hager WD, Barry MB, Wang Y, Kleppinger $\mathrm{A}$, et al. $\mathrm{T}$ cell responses are better correlates of vaccine protection in the elderly. J Immunol. 2006;176(10):6333-9.

47. DiazGranados CA, Dunning AJ, Kimmel M, Kirby D, Treanor J, Collins A, et al. Efficacy of high-dose versus standard-dose influenza vaccine in older adults. N Engl J Med. 2014;371(7):635-45.

48. Wilkinson K, Wei Y, Szwajcer A, Rabbani R, Zarychanski R, Abou-Setta AM, et al. Efficacy and safety of high-dose influenza vaccine in elderly adults: a systematic review and meta-analysis. Vaccine. 2017;35(21):2775-80.

49. Izurieta HS, Thadani N, Shay DK, Lu Y, Maurer A, Foppa IM, et al. Comparative effectiveness of high-dose versus standard-dose influenza vaccines in US residents aged 65 years and older from 2012 to 2013 using Medicare data: a retrospective cohort analysis. Lancet Infect Dis. 2015;15(3):293-300.

50. Shay DK, Chillarige Y, Kelman J, Forshee RA, Foppa IM, Wernecke $\mathrm{M}$, et al. Comparative effectiveness of high-dose versus standard-dose influenza vaccines among US medicare beneficiaries in preventing postinfluenza deaths during 2012-2013 and 2013-2014. J Infect Dis. 2017;215(4):510-7.

51. Young-Xu Y, Van Aalst R, Mahmud SM, Rothman KJ, Thornton Snider J, Westreich D, et al. Relative vaccine effectiveness of high-dose versus standard-dose influenza vaccines among veterans health administration patients. J Infect Dis. 2018;217(11):1718-27.

52. Gravenstein S, Davidson HE, Taljaard M, Ogarek J, Gozalo P, Han L, et al. Comparative effectiveness of high-dose versus standard-dose influenza vaccination on numbers of US nursing home residents admitted to hospital: a cluster-randomised trial. Lancet Respir Med. 2017;5(9):738-46.

53. Becker DL, Chit A, DiazGranados CA, Maschio M, Yau E, Drummond $\mathrm{M}$. High-dose inactivated influenza vaccine is associated with cost savings and better outcomes compared to standard-dose inactivated influenza vaccine in Canadian seniors. Hum Vaccin Immunother. 2016;12(12):3036-42.

54. Chit A, Becker DL, DiazGranados CA, Maschio M, Yau E, Drummond M. Cost-effectiveness of high-dose versus standard-dose inactivated influenza vaccine in adults aged 65 years and older: an economic evaluation of data from a randomised controlled trial. Lancet Infect Dis. 2015;15(12):1459-66.

55. Schaffner W, Chen WH, Hopkins RH, Neuzil K. Effective immunization of older adults against seasonal influenza. Am J Med. 2018;131(8):865-73.

56. Baldo V, Baldovin T, Pellegrini M, Angiolelli G, Majori S, Floreani A, et al. Immunogenicity of three different influenza vaccines against homologous and heterologous strains in nursing home elderly residents. Clin Dev Immunol. 2010;2010:517198.

57. Banzhoff A, Nacci P, Podda A. A new MF59-adjuvanted influenza vaccine enhances the immune response in the elderly with chronic diseases: results from an immunogenicity meta-analysis. Gerontology. 2003;49(3):177-84.

58. Camilloni B, Basileo M, Valente S, Nunzi E, Iorio AM. Immunogenicity of intramuscular MF59-adjuvanted and intradermal administered influenza enhanced vaccines in subjects aged over 60: a literature review. Hum Vaccine Immunother. 2015; 11(3):553-63.

59. Domnich A, Arata L, Amicizia D, Puig-Barbera J, Gasparini R, Panatto D. Effectiveness of MF59-adjuvanted seasonal influenza vaccine in the elderly: a systematic review and meta-analysis. Vaccine. 2017;35(4):513-20.

60. Iob A, Brianti G, Zamparo E, Gallo T. Evidence of increased clinical protection of an MF59-adjuvant influenza vaccine compared to a non-adjuvant vaccine among elderly residents of long-term care facilities in Italy. Epidemiol Infect. 2005;133(4):687-93.

61. Puig-Barbera J, Diez-Domingo J, Varea AB, Chavarri GS, Rodrigo JA, Hoyos SP, et al. Effectiveness of MF59-adjuvanted subunit influenza vaccine in preventing hospitalisations for cardiovascular disease, cerebrovascular disease and pneumonia in the elderly. Vaccine. 2007;25(42):7313-21.

62. Izikson R, Leffell DJ, Bock SA, Patriarca PA, Post P, Dunkle LM, et al. Randomized comparison of the safety of Flublok $((\mathrm{R}))$ versus licensed inactivated influenza vaccine in healthy, medically stable adults $>/=50$ years of age. Vaccine. 2015;33(48):6622-8.

63. Grohskopf LA, Sokolow LZ, Broder KR, Walter EB, Bresee JS, Fry AM, et al. Prevention and control of seasonal influenza with vaccines: recommendations of the advisory committee on immunization practices-United States, 2017-18 influenza season. MMWR Recomm Rep. 2017;66(2):1-20.

64. Dunkle LM, Izikson R, Patriarca P, Goldenthal KL, Muse D, Callahan J, et al. Efficacy of recombinant influenza vaccine in adults 50 years of age or older. N Engl J Med. 2017;376(25):2427-36.

65. Australian Government, Department of Health Influenza: The Australian immunizations handbook. 10th ed. https://immunisati onhandbook.health.gov.au/vaccine-preventable-diseases/influ enza-flu. Accessed 24 Oct 2018.

66. European Centre for Disease Prevention and Control: Seasonal Influenza Vaccines 2018. https://ecdc.europa.eu/en/seasonalinfluenza/prevention-and-control/seasonal-influenza-vaccines. Accessed 11 May 2018.

67. Government of Canada. Canadian Immunization Guide Chapter on Influenza and Statement on Seasonal Influenza Vaccine for 2018-2019. An Advisory Committee Statement (ACS). National Advisory Committee on Immunization (NACI). https://www. canada.ca/en/public-health/services/publications/healthy-living/ canadian-immunization-guide-statement-seasonal-influenza-vacci ne-2018-2019.html\#5.22018. Accessed 11 May 2018.

68. European Centre for Disease Prevention and Control. Types of seasons influenza vaccine 2018. https://ecdc.europa.eu/en/seaso nal-influenza/prevention-and-control/vaccines/types-of-seasonalinfluenza-vaccine. Accessed 11 May 2018. 